\title{
Reduced Order Observer Applied to a Linear Parameter Varying System with Unknown Input
}

\author{
Emanuel Bernardi \\ Facultad Regional San Francisco, \\ Universidad Tecnológica Nacional, \\ Córdoba, Argentina. \\ San Francisco, Av. de la Universidad 501. \\ Email: ebernardi@sanfrancisco.utn.edu.ar
}

\author{
Eduardo J. Adam \\ Facultad de Ingeniería Química, \\ Universidad Nacional del Litoral, \\ Santa Fe, Argentina. \\ Santa Fe, Santiago del Estero 2854. \\ Email: eadam@fiq.unl.edu.ar
}

\begin{abstract}
This paper presents the design of a reduced order observer applied to a Linear Parameter Varying (LPV) system with unknown input. This procedure is employed to develop an actuator Fault Detection and Isolation (FDI) scheme. The observer's design and its stability conditions are guaranteed in terms of a Linear Matrix Inequalities (LMI). Two typical chemical industries processes examples are given to illustrate the use and performance of such approach.
\end{abstract}

Index Terms - state estimation, linear matrix inequalities, fault detection, fault diagnosis, nonlinear systems.

\section{INTRODUCTION}

In the last decades, the Fault Detection and Isolation (FDI) methods, specifically their model-based approaches, have been widely considered. Several contributions and theoretical approaches about its use on linear systems can be found in literature [1]-[5], among others. Besides, there is a considerable amount of articles that involve the development of observers applied to a non-linear system [6]-[8], but this problem is still a challenge due to the trouble of dealing with non-linearities.

Because of the difficulty to design a non-linear observer, many authors prefer to represent these systems by a Linear Parameter Varying (LPV) approach [9], [10]. The idea of this approach is to represent the system as an interpolation of $i$ th affine local models. That is, the interpolation technique presents a good method to schedule a set of linear models by a convex weighting function. The LPV modelling framework is powerful since it allows the application of well-known linear design tools to a wide range of non-linear models.

For the above mentioned reasons, this paper presents the design of a Reduced-order Unknown Input Observer applied to an LPV system (LPV-RUIO). The observer design and its stability conditions are based on the resolution of a Linear Matrix Inequalities (LMI) problem that was solved using MATLAB LMI toolbox [11]. In addition, to perform the states estimation and their consequential residue generation, in order to detect and isolate the actuators faults, the proposed observer is used as part of a bank of dedicated observers.

With the purpose to highlight the behavior of the proposed observer, a numerical simulation of two typical chemical industries processes is given. They are a two tanks non- interacting liquid level process and the highly non-linear Continuous Stirred Tank Reactor (CSTR) process.

This paper is organized as follows. In Section II, the polytopic representation of an affine LPV system is provided. Section III presents the design proposal and stability conditions of an LPV-RUIO. In Section IV a briefly explanation of the detection and isolation methodology is introduced. Subsequently, in Section V and VI, two numerical simulations are provided to show the performance of the proposed approach. Finally, concluding remarks are made in the last section.

\section{LPV SYSTEM REPRESENTATION}

Consider a non-linear system with an unknown input described by the following equation,

$$
\left\{\begin{array}{l}
\dot{x}(t)=f(x(t), u(t), d(t)) \\
y(t)=g(x(t), u(t))
\end{array}\right.
$$

where $x(t) \in \mathbb{R}^{n}, u(t) \in \mathbb{R}^{m}, d(t) \in \mathbb{R}^{q}, y(t) \in \mathbb{R}^{p}$ are the state vector, the known input vector, the unknown input vector and the output vector, respectively.

Under the assumption that functions $f(x(t), u(t), d(t))$ and $g(x(t), u(t))$ are continuously differentiable, it is possible to approximate, or even represent, the dynamic behavior of the system (1) in different operating points through a convex set of $M$ affine models dependent on a variable parameter $\zeta(t)$. That is, the polytopic representation of affine LPV system is,

$$
\begin{aligned}
\dot{x}(t) & =\sum_{i=1}^{M} \mu_{i}(\zeta(t))\left\{A_{i} x(t)+B_{i} u(t)+\Delta x_{i}+D_{i} d(t)\right\} \\
y(t) & =C x(t)
\end{aligned}
$$

where $A_{i}, B_{i}, \Delta x_{i}, D_{i}$ and $C$ are constant matrices of appropriate dimensions, and the weighting functions $\mu_{i}($. depend on a variable parameter $\zeta(t)$ assumed to depend on a measurable variable (inputs or outputs). In addition $\mu_{i}(\zeta(t))$ satisfies,

$$
\mu_{i}(\zeta(t)) \geq 0 ; \quad \sum_{i=1}^{M} \mu_{i}(\zeta(t))=1 .
$$




\section{DESIGN OF THE LPV-RUIO}

Following the development of the observer for linear systems with unknown input introduced by Hou and Muller [1], and under the assumption that rank $D_{i}=q$, a set of nonsingular matrices is selected,

$$
T_{i}=\left[\begin{array}{ll}
N_{i} & D_{i}
\end{array}\right], \quad N_{i} \in \mathbb{R}^{n \times(n-q)} .
$$

Thus, the system (2) is equivalent to,

$$
\begin{aligned}
\dot{\bar{x}}(t) & =\sum_{i=1}^{M} \mu_{i}(\zeta(t))\left\{\bar{A}_{i} \bar{x}(t)+\bar{B}_{i} u(t)+\bar{\Delta}_{i}+\bar{D}_{i} d(t)\right\} \\
y(t) & =\sum_{i=1}^{M} \mu_{i}(\zeta(t))\left\{\bar{C}_{i} \bar{x}(t)\right\}
\end{aligned}
$$

where

$$
\begin{gathered}
x(t)=T_{i} \bar{x}(t)=T_{i}\left[\begin{array}{l}
\bar{x}_{1}(t) \\
\bar{x}_{2}(t)
\end{array}\right] ; \bar{\Delta} x_{i}=T_{i}^{-1} \Delta x_{i}=\left[\begin{array}{c}
\bar{\Delta}_{i_{1}} \\
\bar{\Delta}_{i_{2}}
\end{array}\right] \\
\bar{A}_{i}=T_{i}^{-1} \bar{A}_{i} T_{i}=\left[\begin{array}{ll}
\bar{A}_{i_{11}} & \bar{A}_{i_{12}} \\
\bar{A}_{i_{21}} & \bar{A}_{i_{22}}
\end{array}\right] ; \bar{B}_{i}=T_{i}^{-1} B_{i}=\left[\begin{array}{c}
\bar{B}_{i_{1}} \\
\bar{B}_{i_{2}}
\end{array}\right] \\
\bar{C}_{i}=C T_{i}=\left[C N_{i} C D_{i}\right] ; \quad \bar{D}_{i}=T_{i}^{-1} D_{i}=\left[\begin{array}{c}
0 \\
I_{q}
\end{array}\right]
\end{gathered}
$$

with $\bar{x}_{1}(t) \in \mathbb{R}^{n-q}, \bar{x}_{2}(t) \in \mathbb{R}^{q}$.

Because of (6) it is observed that (5a) involves directly the unknown input in the state $\bar{x}_{2}(t)$. Then, it is possible to drop this state and rewrite the system (5) without the unknown input as,

$$
\begin{aligned}
{\left[\begin{array}{ll}
I_{n-q} \quad 0
\end{array}\right] \dot{\bar{x}}(t)=} & \sum_{i=1}^{M} \mu_{i}(\zeta(t))\left\{\left[\begin{array}{ll}
\bar{A}_{i_{11}} & \bar{A}_{i_{12}}
\end{array}\right] \bar{x}(t)\right. \\
& \left.+\bar{B}_{i_{1}} u(t)+\bar{\Delta} x_{i_{1}}\right\} \\
y(t)= & \sum_{i=1}^{M} \mu_{i}(\zeta(t))\left\{\left[C N_{i} C D_{i}\right] \bar{x}(t)\right\} .
\end{aligned}
$$

Assuming that $\bar{x}_{2}(t)$ is obtained from $y(t)$ and, if the matrix $\left[C D_{i}\right]$ has full column rank, then there exists a non-singular matrix

$$
U_{i}=\left[\begin{array}{ll}
C D_{i} & Q_{i}
\end{array}\right], \quad Q_{i} \in \mathbb{R}^{n \times(n-q)}
$$

being

$$
U_{i}^{-1}=\left[\begin{array}{c}
U_{i_{1}} \\
U_{i_{2}}
\end{array}\right], \quad U_{i_{1}} \in \mathbb{R}^{q \times n}, U_{i_{2}} \in \mathbb{R}^{(n-q) \times n}
$$

multiplying both sides of (7b) by $U_{i}^{-1}$ and isolating,

$$
\begin{aligned}
\bar{x}_{2}(t) & =\sum_{i=1}^{M} \mu_{i}(\zeta(t))\left\{U_{i_{1}} y(t)-U_{i_{1}} C N_{i} \bar{x}_{1}(t)\right\} \\
y(t) & =\sum_{i=1}^{M} \mu_{i}(\zeta(t))\left\{C N_{i} \bar{x}_{1}(t)\right\}
\end{aligned}
$$

afterwards, substituting (10a) into (7a) and combining it with (10b) derive in (2) transform to,

$$
\begin{aligned}
\dot{\bar{x}}_{1}(t)= & \sum_{i=1}^{M} \mu_{i}(\zeta(t))\left\{\tilde{A}_{i_{1}} \bar{x}_{1}(t)+E_{i_{1}} y(t)\right. \\
& \left.+\bar{B}_{i_{1}} u(t)+\bar{\Delta} x_{i_{1}}\right\} \\
y(t)= & \sum_{i=1}^{M} \mu_{i}(\zeta(t))\left\{\tilde{C}_{i_{1}} \bar{x}_{1}(t)\right\}
\end{aligned}
$$

where $\tilde{C}_{i_{1}}=C N_{i}, \tilde{A}_{i_{1}}=\bar{A}_{i_{11}}-\bar{A}_{i_{12}} U_{i_{1}} C N_{i}$ and $E_{i_{1}}=$ $\bar{A}_{i_{12}} U_{i_{1}}$.

At this point, if the pair $\left\{\tilde{A}_{i_{1}}, \tilde{C}_{i_{1}}\right\}$ is observable, following the conventional Luenberger observer design procedure [12], it is possible to design a reduced-order observer for a system free of unknown inputs (11) as,

$$
\dot{\Phi}(t)=\sum_{i=1}^{M} \mu_{i}(\zeta(t))\left\{K_{i} \Phi(t)+\bar{B}_{i_{1}} u(t)+\bar{\Delta}^{-} x_{i_{1}}+L_{i}^{*} y(t)\right\}
$$

with $\Phi(t) \in \mathbb{R}^{(n-q)}, L_{i}^{*}=L_{i}+E_{i_{1}}$ and $K_{i}=\tilde{A}_{i_{1}}-L_{i} \tilde{C}_{i_{1}}$. Where $L_{i} \in \mathbb{R}^{(n-q) \times(p-q)}$ is the gain of the observer to be designed.

For that, the state estimation error is defined as,

$$
e(t)=\bar{x}_{1}(t)-\Phi(t)
$$

therefore, the estimation error dynamics is

$$
\begin{aligned}
\dot{e}(t) & =\dot{\bar{x}}_{1}(t)-\dot{\Phi}(t) \\
& =\sum_{i=1}^{M} \mu_{i}(\zeta(t))\left\{\tilde{A}_{i_{1}} \bar{x}_{1}(t)-K_{i} \Phi(t)-L_{i} y(t)\right\} \\
& =\sum_{i=1}^{M} \mu_{i}(\zeta(t))\left\{K_{i}\left(\bar{x}_{1}(t)-\Phi(t)\right)\right\}
\end{aligned}
$$

becomes,

$$
\dot{e}(t)=\sum_{i=1}^{M} \mu_{i}(\zeta(t)) K_{i} e(t)
$$

in this way, if $K_{i}$ is Hurwitz, $\dot{e}(t) \rightarrow 0$ asymptotically.

Theorem 1: If there exists a symmetric matrix $X \succ 0$ and $W_{i}$, such that the following conditions hold $\forall i \in$ $\{1,2,3, \ldots, M\}$ :

$$
\left(X \tilde{A}_{i_{1}}-W_{i} \tilde{C}_{i_{1}}\right)^{T}+\left(X \tilde{A}_{i_{1}}-W_{i} \tilde{C}_{i_{1}}\right)+2 \alpha X \prec 0
$$

then the observer (12) is a Linear Parameter Varying Reducedorder Unknown Input Observer (LPV-RUIO). That is, $e(t)$ towards zero asymptotically for any initial state $e(0)$.

Proof: From (13), and choosing a Lyapunov function with a symmetric matrix $X \succ 0$.

$$
V(t)=e(t)^{T} X e(t)
$$

thus, the exponential convergence of the estimation error is guaranteed if,

$$
\dot{V}(t)+2 \alpha V(t)<0
$$


where $\alpha$ is the decay rate constant [13]. Then, using (15) and (17) it is obtained,

$$
\begin{aligned}
\dot{V}(t)= & \dot{e}^{T}(t) X e(t)+e(t) X \dot{e}^{T}(t) \\
= & \sum_{i=1}^{M} \mu_{i}(\zeta(t)) e^{T}(t) K_{i}^{T} X e(t) \\
& +\sum_{i=1}^{M} \mu_{i}(\zeta(t)) e^{T}(t) X K_{i} e(t) \\
= & \sum_{i=1}^{M} \mu_{i}(\zeta(t))\left\{e^{T}(t)\left(K_{i}^{T} X+X K_{i}\right) e(t)\right\}
\end{aligned}
$$

hence, replacing (17) and (19) in (18) it results,

$$
\sum_{i=1}^{M} \mu_{i}(\zeta(t))\left\{e^{T}(t)\left(K_{i}^{T} X+X K_{i}+2 \alpha X\right) e(t)\right\}<0
$$

notice that $\left(K_{i}^{T} X+X K_{i}+2 \alpha X\right) \prec 0, \forall_{i} \in\{1,2,3, \ldots, M\}$, implies that $e(t)$ towards zero asymptotically for any initial state $e(0)$.

As defined $K_{i}=\tilde{A}_{i_{1}}-L_{i} \tilde{C}_{i_{1}}$. Therefore, replacing the previous inequality,

$$
\begin{gathered}
\left(\tilde{A}_{i_{1}}-L_{i} \tilde{C}_{i_{1}}\right)^{T} X+X\left(\tilde{A}_{i_{1}}-L_{i} \tilde{C}_{i_{1}}\right)+2 \alpha X \prec 0 \\
\tilde{A}_{i_{1}}^{T} X-\tilde{C}_{i_{1}}^{T} L_{i}^{T} X+X \tilde{A}_{i_{1}}-X L_{i} \tilde{C}_{i_{1}}+2 \alpha X \prec 0 .
\end{gathered}
$$

Moreover, to eliminate the non-existing linearities, it is defined $W_{i}=X L_{i}$. Resulting,

$$
\left(X \tilde{A}_{i_{1}}-W_{i} \tilde{C}_{i_{1}}\right)^{T}+\left(X \tilde{A}_{i_{1}}-W_{i} \tilde{C}_{i_{1}}\right)+2 \alpha X \prec 0
$$

Remark 1: The theorem 1 shows that the LPV-RUIO design is solved through the LMI (16). For that, the LMI Lab [11] package of MATLAB software is used.

Thereby, from (12), with $\Phi(t) \rightarrow \hat{\bar{x}}_{1}(t)$ according to $t \rightarrow$ $\infty$, getting

$$
\begin{aligned}
\hat{x} & =\sum_{i=1}^{M} \mu_{i}(\zeta(t)) T_{i} \hat{\bar{x}} \\
& =\sum_{i=1}^{M} \mu_{i}(\zeta(t)) T_{i}\left[\begin{array}{c}
\Phi(t) \\
U_{i_{1}} y(t)-U_{i_{1}} C N_{i} \Phi(t)
\end{array}\right]
\end{aligned}
$$

with $\hat{x}(t) \rightarrow x(t)$, according to $t \rightarrow \infty$.

\section{Actuator Fault Detection using LPV-RUiO}

If the LPV-RUIO given by (12) can be designed such that $e(t)$ tends to zero when there is no fault and defining $r(t)=$ $|\hat{y}(t)-y(t)|=|C e(t)|$, then $r(t)$ also tends to zero when the system is fault free. Based on this observation and following the classical FDI schemes [3], the fault detection is achieved as:

$$
\text { Fault }= \begin{cases}\text { False } & \text { if } r(t) \leq r_{t h} \\ \text { True } & \text { if } r(t)>r_{t h}\end{cases}
$$

where $r_{t h}$ is a fixed detection threshold. Besides, according to the existing schemes ([4], [5]) it is possible to construct a bank of observers and from analyzing their residues the actuator faults can be isolated.

\section{ILLUSTRATIVE EXAMPLE I}

Consider the model of a two tanks non-interacting liquid level process, represented in Fig. 1, its physical and operational parameter values appear in Table I.

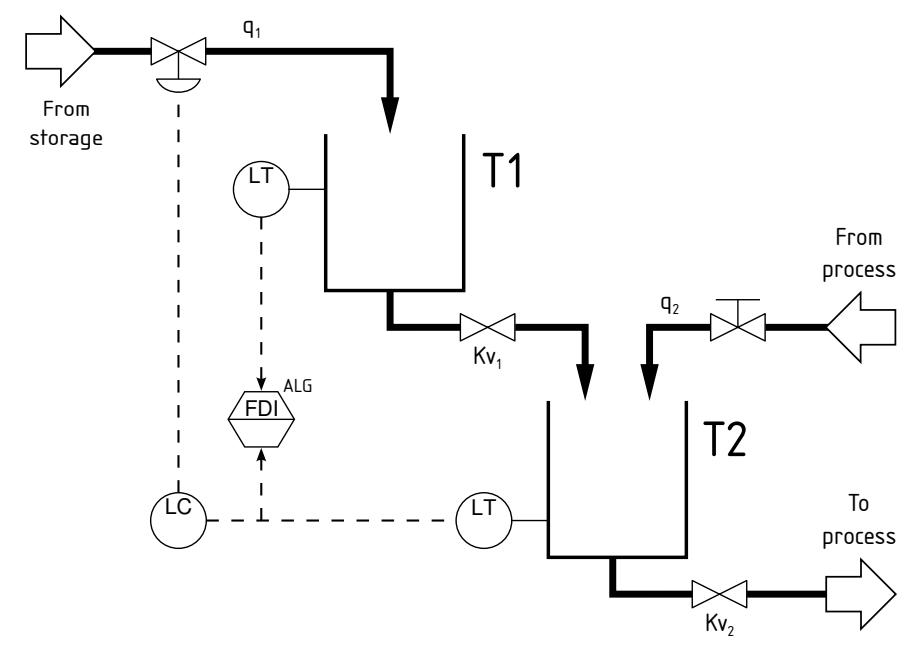

Fig. 1. Diagram of a two tanks non-interacting process.

TABLE I

SYSTEM PARAMETERS OF TWO TANKS NON-INTERACTING PROCESS

\begin{tabular}{lll}
\hline Parameter & Description & Value \\
\hline$h_{1_{\max }}, h_{2_{\max }}$ & Maximum height & $0.6 \mathrm{~m}$ \\
$d_{1}, d_{2}$ & Tanks diameter & $0.04 \mathrm{~m}$ \\
$q_{1_{\max }}, q_{2 \max }$ & Maximum flow rate & $61 \mathrm{~min}-1$ \\
$K_{v_{1}}$ & Flow coefficient 1 & $0.9 \times 10^{-4} \mathrm{~m}^{3} \mathrm{~s}^{-1} \mathrm{bar}^{-1}$ \\
$K_{v_{2}}$ & Flow coefficient 2 & $1 \times 10^{-4} \mathrm{~m}^{3} \mathrm{~s}^{-1} \mathrm{bar}^{-1}$ \\
\hline
\end{tabular}

The non-linear model equations of the two tanks noninteracting liquid level process are defined as:

$$
\begin{aligned}
& A_{1} \frac{d h_{1}(t)}{d t}=q_{1}(t)-K_{v_{1}} \sqrt{h_{1}(t)} \\
& A_{2} \frac{d h_{2}(t)}{d t}=q_{2}(t)+K_{v_{1}} \sqrt{h_{1}(t)}-K_{v_{2}} \sqrt{h_{2}(t)} .
\end{aligned}
$$

Finally, according to Fig. 1, the state variable $h_{2}$ is controlled by the storage feed flow rate $q_{1}$ and the process flow rate $q_{2}$.

\section{A. LPV-RUIO Design}

Using the Parameterized Jacobian Linearization (PJL) technique [9], it is possible to rewrite (25) like an LPV model. For that, the variable parameters vector, with dimension $N=2$, was defined depending on the outputs, $\zeta(t):=\left[\begin{array}{ll}h_{1}(t) & h_{2}(t)\end{array}\right]$. To obtain a proper representation of the non-linear system, $L=3$ linearization points per parameter were used. That is,

$$
\begin{aligned}
\rho_{j, 1} & =\min \left\{\zeta_{j}(t)\right\} \\
\rho_{j, 2} & =\operatorname{mid}\left\{\zeta_{j}(t)\right\} \\
\rho_{j, 3} & =\max \left\{\zeta_{j}(t)\right\}
\end{aligned}
$$


with $j \in[1, \ldots, N]$ and $k \in[1, \ldots, L]$. As a result, we get

$$
\begin{aligned}
& A_{i}=\left[\begin{array}{cc}
-\frac{K_{v_{1}}}{2 A_{1} \sqrt{h_{1_{i}}}} & 0 \\
\frac{K_{v_{1}}}{2 A_{2} \sqrt{h_{1_{i}}}} & -\frac{K_{v_{2}}}{2 A_{2} \sqrt{h_{2_{i}}}}
\end{array}\right] \\
& B_{i}=\left[\begin{array}{cc}
\frac{1}{A_{1}} & 0 \\
0 & \frac{1}{A_{2}}
\end{array}\right], \quad C=\left[\begin{array}{ll}
1 & 0 \\
0 & 1
\end{array}\right]
\end{aligned}
$$

and $\Delta x_{i}=x_{i}-\left\{A_{i} x_{i}+B_{i} u_{i}\right\}$, where the variable indicated with a subscript $i$ corresponds to its value at the $i$ th linearization point. Next, we obtain membership functions of each parameter

$$
\begin{aligned}
M_{j, 1}\left(\zeta_{j}(t)\right) & =\frac{\rho_{j, 1}-\zeta_{j}(t)}{\rho_{j, 2}-\rho_{j, 1}} \\
M_{j, 2}\left(\zeta_{j}(t)\right) & =\frac{\rho_{j, 2}-\zeta_{j}(t)}{\rho_{j, 1}-\rho_{j, 3}} \\
M_{j, 3}\left(\zeta_{j}(t)\right) & =\frac{\zeta_{j}(t)-\rho_{j, 3}}{\rho_{j, 3}-\rho_{j, 2}} .
\end{aligned}
$$

Taking everything into account, the $M=L^{N}=9$ weighting functions corresponding to each lineal model was defined

$$
\begin{aligned}
& \mu_{1}(\zeta(t))=M_{1,1}\left(\zeta_{1}(t)\right) M_{2,1}\left(\zeta_{2}(t)\right) \\
& \mu_{2}(\zeta(t))=M_{1,1}\left(\zeta_{1}(t)\right) M_{2,2}\left(\zeta_{2}(t)\right) \\
& \mu_{3}(\zeta(t))=M_{1,1}\left(\zeta_{1}(t)\right) M_{2,3}\left(\zeta_{2}(t)\right) \\
& \mu_{4}(\zeta(t))=M_{1,2}\left(\zeta_{1}(t)\right) M_{2,1}\left(\zeta_{2}(t)\right) \\
& \mu_{5}(\zeta(t))=M_{1,2}\left(\zeta_{1}(t)\right) M_{2,2}\left(\zeta_{2}(t)\right) \\
& \mu_{6}(\zeta(t))=M_{1,2}\left(\zeta_{1}(t)\right) M_{2,3}\left(\zeta_{2}(t)\right) \\
& \mu_{7}(\zeta(t))=M_{1,3}\left(\zeta_{1}(t)\right) M_{2,1}\left(\zeta_{2}(t)\right) \\
& \mu_{8}(\zeta(t))=M_{1,3}\left(\zeta_{1}(t)\right) M_{2,2}\left(\zeta_{2}(t)\right) \\
& \mu_{9}(\zeta(t))=M_{1,3}\left(\zeta_{1}(t)\right) M_{2,3}\left(\zeta_{2}(t)\right)
\end{aligned}
$$

At last, defining $D_{i}=\left[\begin{array}{ll}\frac{1}{A_{1}} & 0\end{array}\right]^{T}$ and solving the LMI (16), we get the matrices to construct the observer (12) that was used to detect and isolate the failure of the valve $q_{2}$. On the other hand, defining $D_{i}=\left[\begin{array}{ll}0 & \frac{1}{A_{2}}\end{array}\right]^{T}$, and repeating the previous procedure, we construct the observer to detect and isolate the failure of the valve $q_{1}$.

\section{B. Numerical Simulation}

To evaluate the performance and effectiveness of the proposed LPV-RUIO, the presented FDI scheme was simulated on the system (25). Firstly, two set-point step changes from the initial condition $h_{2}=0.4 \mathrm{~m}$ were applied $h_{2}=0.3 \mathrm{~m}$ at $t=200 \mathrm{~s}$ and $h_{2}=0.25 \mathrm{~m}$ at $t=400 \mathrm{~s}$ to show the observer state estimation tracking capacity. Secondly, between $t=400 \mathrm{~s}$ and $t=900 \mathrm{~s}$ an incipient gain degradation up to $10 \%$ from $q_{1_{\max }}$ flow rate is introduced. Thirdly, an abrupt fault with $10 \%$ from $q_{2_{\max }}$ magnitude, occurred on valve $q_{2}$ between $t=1000 \mathrm{~s}$ and $t=1500 \mathrm{~s}$. Finally, at $t>1200 \mathrm{~s}$ an abrupt fault occurred on valve $q_{1}$ with a magnitude of $10 \%$ from $q_{1_{\max }}$ was injected.

It is important to note that to build a more realistic simulation, a white measurement noise was added.
The proposed observer and the non-linear system (25) outputs are depicted in the Fig. 2. As can be seen, the performance and tracking capacity exhibited in the fault-free operation mode is correct. Besides, the Fig. 3 shows the residues obtained from the difference between the real and estimate states, which, when comparing this value with a constant threshold, according to (24), it is possible to appreciate the fault occurrence. At last, the Fig. 4 exhibits the behavior of the weighting functions.
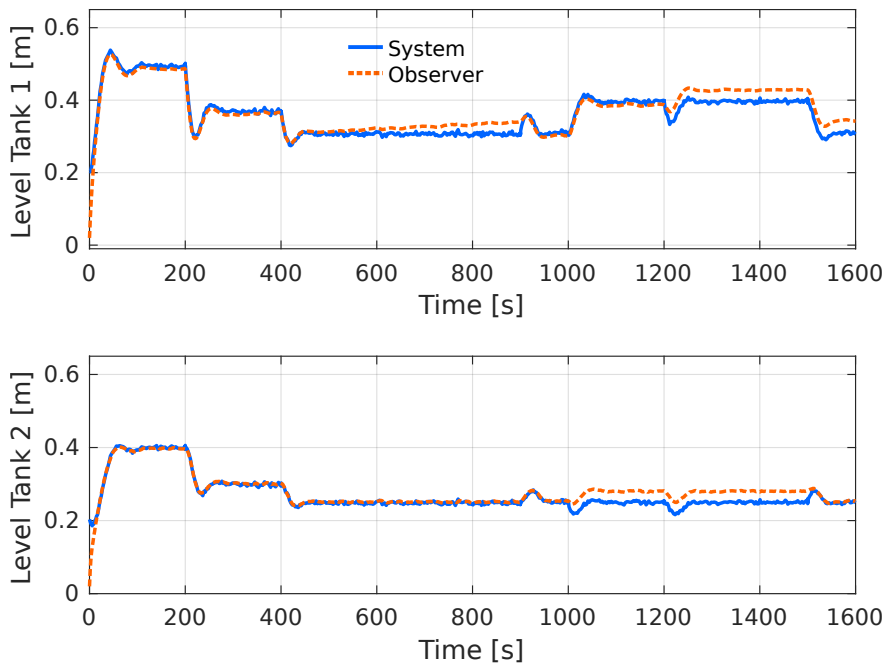

Fig. 2. Liquid levels.
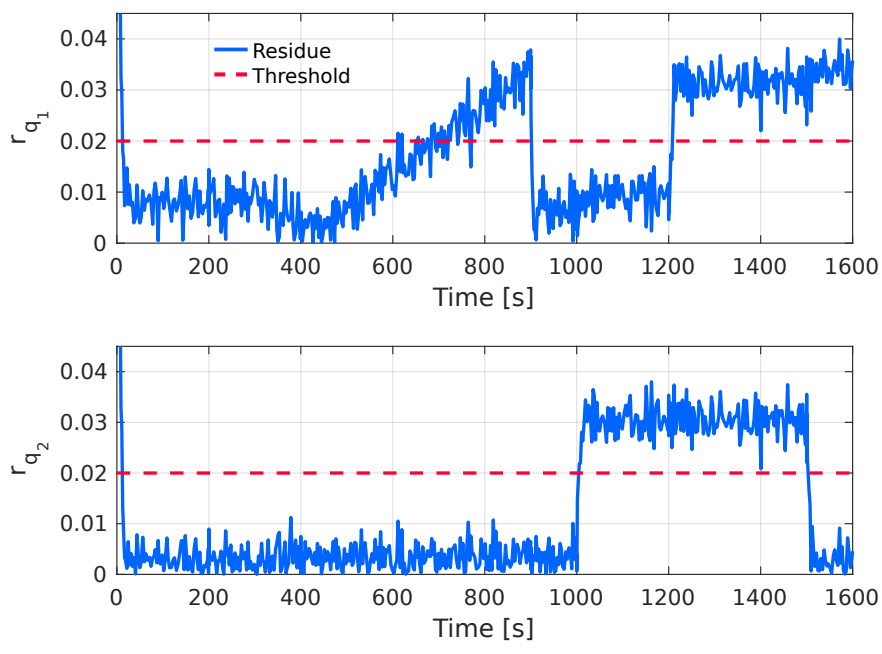

Fig. 3. Residuals.

\section{ILlustrative EXAMPLE II}

Consider the model of a CSTR process, represented in Fig. 5, its physical and operational parameter values appear in Table II. This model is a modified version of the CSTR example presented by Morningred et al. [14]. In the original model, the system operates to constant volume.

Therefore, the CSTR process consists of an irreversible, exothermic reaction, $A \rightarrow B$, in a variable volume reactor 


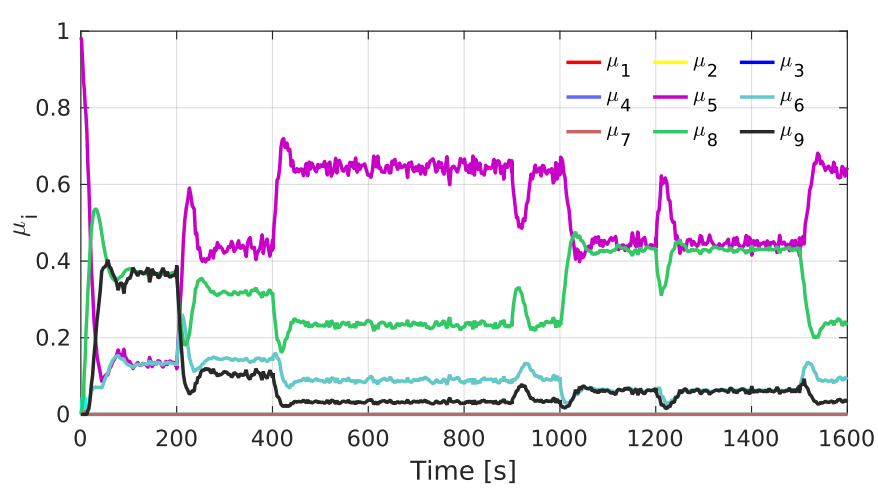

Fig. 4. Weighting functions.

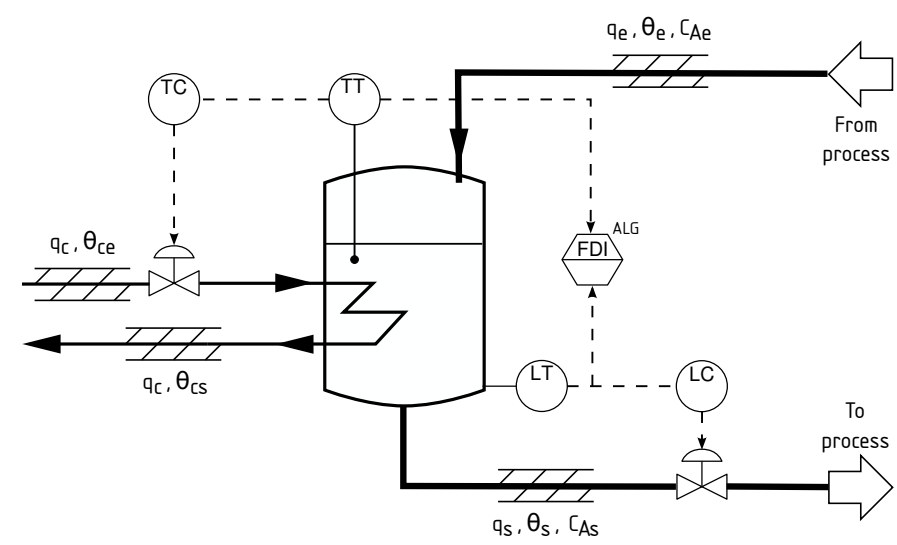

Fig. 5. Diagram of a Continuous Stirred Tank Reactor process.

cooled by a single coolant stream which can be modeled by the following equations:

$$
\begin{aligned}
\frac{d V(t)}{d t}= & q_{e}-q_{s}(t) \\
\frac{d C_{A}(t)}{d t}= & \frac{q_{e}}{V(t)}\left(C_{A e}-C_{A}(t)\right)-k_{0} e^{\frac{-E}{R T(t)}} C_{A}(t) \\
\frac{d T(t)}{d t}= & \frac{q_{e}}{V(t)}\left(T_{e}-T(t)\right)-k_{1} e^{\frac{-E}{R T(t)}} C_{A}(t) \\
& +\frac{q_{c}(t)}{V(t)} k_{2}\left(1-e^{\frac{-k_{3}}{q_{c}(t)}}\right)\left(T_{c e}-T(t)\right)
\end{aligned}
$$

TABLE II

SYSTEM PARAMETERS OF CSTR PROCESS.

\begin{tabular}{lll}
\hline Parameter & Description & Value \\
\hline$q_{e}$ & Feed flow rate & $100 \mathrm{~min}^{-1}$ \\
$T_{e}$ & Feed temperature & $350 \mathrm{~K}$ \\
$C_{A e}$ & Feed concentration & $1 \mathrm{~mol} \mathrm{l}^{-1}$ \\
$T_{c e}$ & Inlet coolant temperature & $350 \mathrm{~K}$ \\
$E / R$ & Activation energy term & $1 \times 10^{4} \mathrm{~K}$ \\
$\Delta \mathrm{H}$ & Heat of reaction & $-2 \times 10^{5} \mathrm{cal} \mathrm{mol}^{-1}$ \\
$C_{p}, C_{p c}$ & Specific heats & $1 \mathrm{cal} \mathrm{g}^{-1} \mathrm{~K}$ \\
$\rho, \rho_{c}$ & Liquid densities & $1 \times 10^{3} \mathrm{gl}^{-1}$ \\
$h_{A}$ & Heat transfer term & $7 \times 10^{5} \mathrm{cal} \mathrm{min}^{-1} \mathrm{~K}$ \\
$k_{0}$ & Reaction rate constant & $7.2 \times 10^{10} \mathrm{~min}^{-1}$ \\
$q_{s_{\max }}$ & Maximum output flow rate & $1101 \mathrm{~min}^{-1}$ \\
$q_{c_{\max }}$ & Maximum coolant flow rate & $1101 \mathrm{~min}^{-1}$ \\
\hline & &
\end{tabular}

where

$$
k_{1}=\frac{\Delta H k_{0}}{\rho C_{p}}, k_{2}=\frac{\rho C_{p c}}{\rho_{c} C_{p}}, k_{3}=\frac{h_{A}}{\rho_{c} C_{p c}} .
$$

Finally, according to Fig. 5, the state variables $V$ and $C_{A}$ are controlled by the process flow rate $q_{s}$ and the coolant flow rate $q_{c}$, respectively. It should be clarified that the state variable $C_{A}$ is controlled indirectly from the state $T$.

\section{A. LPV-RUIO Design}

Following the design procedure used in the previous example, we defined the variables parameters vector, with dimension $N=2$, like $\zeta(t):=\left[\begin{array}{ll}V(t) & T(t)\end{array}\right]$, and then we performed the PJL in $L=3$ points per parameter. That is,

$$
\begin{aligned}
& A_{i}=\left[\begin{array}{ccc}
0 & 0 & 0 \\
\frac{q_{e}\left(C_{A_{i}}-C_{A e}\right)}{V_{i}^{2}} & -\frac{q_{e}}{V_{i}}-k_{0} e^{\frac{-E}{R T_{i}}} & -\frac{E C_{A_{i}} k_{0} e^{\frac{-E}{R T_{i}}}}{R T_{i}^{2}} \\
A_{31} & -k_{1} e^{\frac{-E}{R T_{i}}} & A_{33}
\end{array}\right] \\
& B_{i}=\left[\begin{array}{cc}
-1 & 0 \\
0 & 0 \\
0 & B_{32}
\end{array}\right], \quad C=\left[\begin{array}{lll}
1 & 0 & 0 \\
0 & 1 & 0 \\
0 & 0 & 1
\end{array}\right]
\end{aligned}
$$

and $\Delta x_{i}=x_{i}-\left\{A_{i} x_{i}+B_{i} u_{i}\right\}$, where

$$
\begin{aligned}
& A_{31}=\frac{q_{e}\left(T_{i}-T_{e}\right)}{V_{i}^{2}}-\frac{k_{2} q_{c_{i}}\left(e^{\frac{-k_{3}}{q_{c_{i}}}}-1\right)\left(T_{i}-T_{c e}\right)}{V_{i}^{2}} \\
& A_{33}=\frac{k_{2} q_{c_{i}}\left(e^{\frac{-k_{3}}{q_{c_{i}}}}-1\right)}{V_{i}}-\frac{q_{e}}{V_{i}}-\frac{E C_{A_{i}} k_{1} e^{\frac{-E}{R T_{i}}}}{R T_{i}^{2}} \\
& B_{32}=\frac{k_{2}\left(T_{i}-T_{c e}\right)\left(e^{\frac{-k_{3}}{q_{c_{i}}}}-1\right)}{V_{i}}+\frac{k_{2} k_{3}\left(T_{i}-T_{c e}\right) e^{\frac{-k_{3}}{q_{c_{i}}}}}{V_{i}} .
\end{aligned}
$$

At last, defining $D_{i}=\left[\begin{array}{lll}-1 & 0 & 0\end{array}\right]^{T}$ and solving the LMI (16), we get the matrices to construct the observer (12) that was used to detect and isolate the failure of the valve $q_{s}$. On the other hand, defining $D_{i}=\left[\begin{array}{lll}0 & 0 & B_{32}\end{array}\right]^{T}$, and repeating the previous procedure, we construct the observer to detect and isolate the failure of the valve $q_{c}$.

\section{B. Numerical Simulation}

Again, following the procedure of the previous example to evaluate the performance and effectiveness of the proposed LPV-RUIO, the presented FDI scheme was simulated on the system (30). Firstly, three set-point changes from the initial condition $C_{A}=0.9 \mathrm{moll}^{-1}$ and $V=100 \mathrm{l}$ were applied $C_{A}=0.12 \mathrm{moll}^{-1}$ at $t=25 \mathrm{~s}, C_{A}=0.7 \mathrm{moll}^{-1}$ at $t=50 \mathrm{~s}$, $V=98 \mathrm{l}$ at $t=100 \mathrm{~s}$ and $V=102 \mathrm{l}$ at $t=250 \mathrm{~s}$ to show the observer state estimation tracking. Secondly, between $t=50 \mathrm{~s}$ and $t=150 \mathrm{~s}$, it is introduced an incipient gain degradation up to $5 \%$ from $q_{s_{\max }}$. Thirdly, an abrupt fault, with magnitude of $5 \%$ from $q_{s_{\max }}$, occurred on valve $q_{s}$ between $t=200 \mathrm{~s}$ and $t=350 \mathrm{~s}$. Finally, at $t>250 \mathrm{~s}$ an abrupt fault occurred on valve $q_{c}$ with a magnitude of $5 \%$ from $q_{c_{\max }}$ was injected. Adding a white measurement noise as well. 
The proposed observer and the non-linear system (30) are depicted in the Fig. 6. Besides the Fig. 7 shows the fault occurrence. At last, the Fig. 8 exhibits the behavior of the weighting functions.
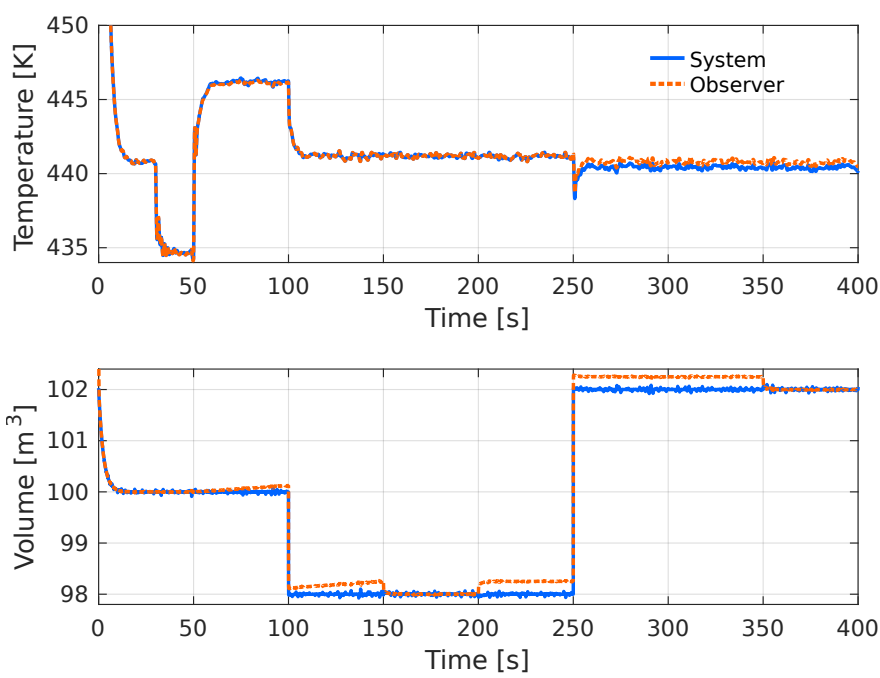

Fig. 6. Volume and Temperature.
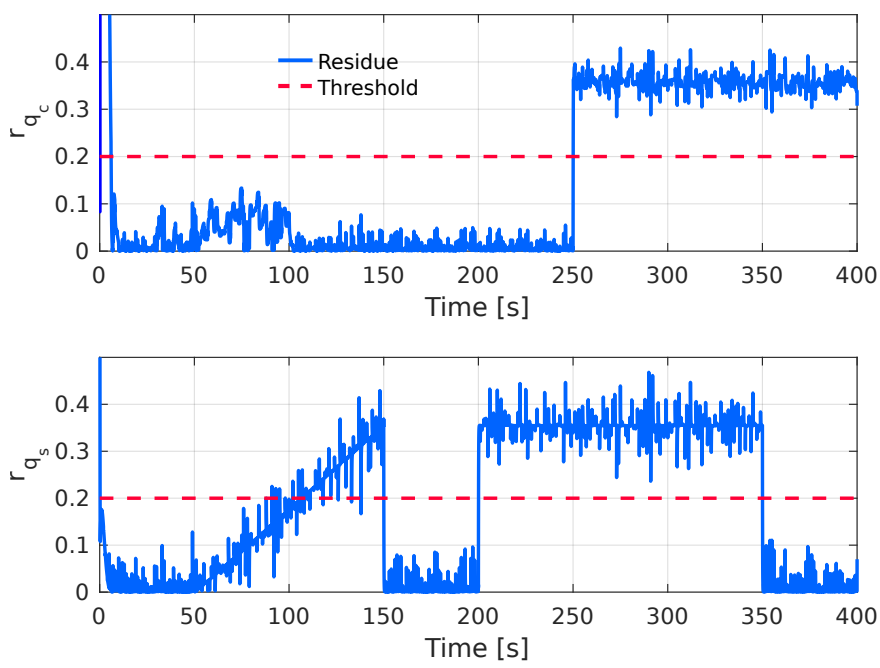

Fig. 7. Residuals.

\section{CONCLUSION}

In this paper, it is presented an FDI scheme designed using a reduced order observer applied to an LPV system with unknown input. The proposed observer and its stability conditions are based on the resolution of an LMI problem that had been performed using MATLAB LMI toolbox.

It is important to note that the main proposal of this work is the design of an LPV-RUIO from the linear-like design tools and the proposition of an LMI problem. Therefore, it allows the development of a particular observer bank to diagnose and identify specific faults.

In addition, with the purpose to highlight the behavior of the proposed observer, a numerical simulation of two typical

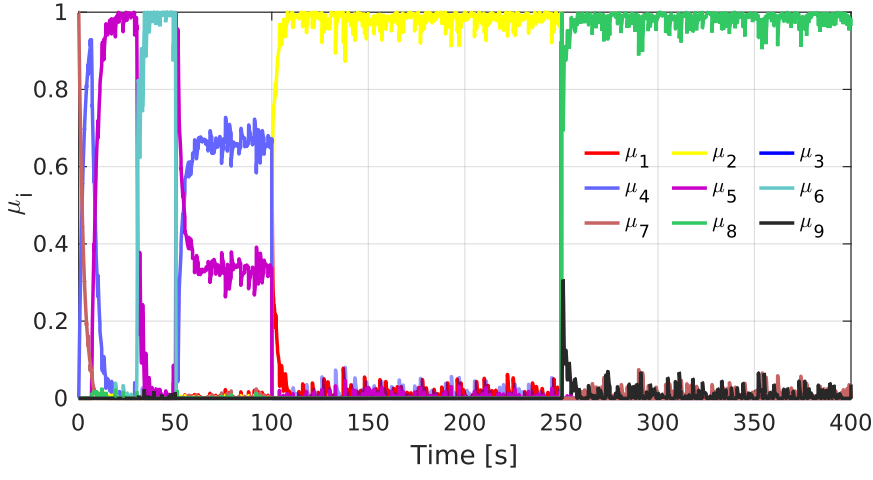

Fig. 8. Weighting functions.

chemical industries processes were given. Consequently, the simulation results confirm the robustness and effectiveness of the proposed scheme for the actuator fault detection over a non-linear system in the presence of external disturbance.

Based on the results of the paper, interesting future studies may extend the proposed technique to develop a fault estimation schemes or even a fault tolerant control system.

\section{ACKNOWLEDGMENTS}

The first author would like to thank UTN for the grant support through its Doctoral Scholarship Program.

\section{REFERENCES}

[1] M. Hou and P. C. Muller, "Design of observers for linear systems with unknown inputs," IEEE Transactions on Automatic Control, vol. 37, no. 6, pp. 871-875, 061992.

[2] M. Darouach, M. Zasadzinski, and S. J. Xu, "Full-order observers for linear systems with unknown inputs," IEEE transactions on automatic control, vol. 39, no. 3, pp. 606-609, 1994.

[3] J. Chen and R. J. Patton, Robust Model-Based Fault Diagnosis for Dynamic Systems, 1st ed. Kluwer Academic Publishers, 1999.

[4] R. Isermann, Fault-Diagnosis Systems: an Introduction from Fault Detection to Fault Tolerance, 1st ed. Springer Science \& Business Media, 2006.

[5] M. F. Pico and E. J. Adam, "Fault diagnosis and tolerant control using observer banks applied to continuous stirred tank reactor," Advances in Science, Technology and Engineering Systems Journal, vol. 2, no. 3, pp. 171-181, 2017.

[6] W. Chen and M. Saif, "Unknown input observer design for a class of nonlinear systems: an lmi approach," 2006 American Control Conference, no. 1, pp. 834-838, 2006.

[7] J. Korbicz and M. Witczak, "An extended unknown input observer-based approach to fault diagnosis of a two-tank system," IFAC Proceedings Volumes (IFAC-PapersOnline), vol. 7, no. PART 1, pp. 922-927, 2007.

[8] W. Chen and M. Saif, "Design of a ts based fuzzy nonlinear unknown input observer with fault diagnosis applications," in 2007 American Control Conference, July 2007, pp. 2545-2550.

[9] C. Briat, "Commande et observation robustes des systemes lpv retardés," Ph.D. dissertation, Grenoble INP, 2008.

[10] J. S. Shamma, "An overview of lpv systems," in Control of linear parameter varying systems with applications. Springer, 2012, pp. 3-26.

[11] P. Gahinet, A. Nemirovski, A. J. Laub, and M. Chilali, LMI Control Toolbox User's Guide. MathWorks, 051995.

[12] D. G. Luenberger, "Observing the State of a Linear System," IEEE Transactions on Military Electronics, vol. 8, no. 2, pp. 74-80, 1964.

[13] S. P. Boyd, L. E. Ghaoui, E. Feron, and V. Balakrishnan, Linear Matrix Inequalities in System and Control Theory. Society for Industrial and Applied Mathematics, 1994, vol. 15.

[14] J. D. Morningred, B. E. Paden, D. E. Seborg, and D. A. Mellichampt, "An Adaptive Nonlinear Predictive Controller," Chemical Engineering Science, vol. 47, no. 4, pp. 755-762, 1992. 